\title{
CdSe/ZnSe Quantum Dots Formed by Low Temperature Epitaxy and In-Situ Annealing: Properties and Growth Optimization
}

\author{
S. Mahapatra, C. Schumacher, T. Kiessling, \\ G.V. Astakhov, U. Bass, W. Ossau, J. Geurts
}

AND K. BRUNNER

Universität Würzburg, EP III, Physikalisches Institut am Hubland, 97074 Würzburg, Germany

\begin{abstract}
The formation of CdSe/ZnSe quantum dots by a method combining a low temperature MBE growth of a CdSe layer and its subsequent in-situ annealing at temperatures between $280-340^{\circ} \mathrm{C}$ has been studied. The thermal treatment results in a re-organization of the surface from a nearly two-dimensional layer to an ensemble of three-dimensional dot-like features. In this work we optimized the different growth and annealing parameters of this process and compared the properties of the resultant dots with those of dots grown by conventional $\mathrm{MBE}$ at $300^{\circ} \mathrm{C}$. It is demonstrated that the luminescence properties of the dots for both growth techniques are comparable but the areal density achieved by the in-situ annealing technique is an order of magnitude lower. From high resolution X-ray diffraction results, it could be established that no desorption takes place despite significantly long annealing duration. Beyond a nominal coverage of 3.5 ML CdSe, stacking faults are generated, leading to a gradual decrease in luminescence intensities and an overlap of pendellösung fringes in X-ray diffractograms.
\end{abstract}

PACS numbers: 81.16.Dn, 81.05.Dz, 73.21.La

\section{Introduction}

Self-assembled CdSe quantum dots (QDs) in ZnSe based matrices have been studied extensively due to their strong quantum and nonlinear photonic effects, which can lead to novel optoelectronic applications in the much covered green to blue spectral range. A viable method for the formation of QDs relies 
on the Stranski-Krastanow (S-K) morphology transition, wherein self-organized, nanometer-sized, coherent islands are formed as a consequence of elastic strain relaxation during heteroepitaxy. Although well-known for several semiconductor systems, the transition is not so clearly established for the CdSe-ZnSe system, possibly due to energetically competitive alternative routes of strain relaxation, like intermixing at typical growth conditions [1] and defect generation [2]. Efforts to grow CdSe dots by conventional MBE at a temperature in the range of $280-350^{\circ} \mathrm{C}$ result in the formation of no well-defined 3D QD like features but a random fluctuation in thickness and/or composition. This leads to a very high density of Cd-rich regions showing a strong vertical but weak in-plane confinement, and possibly coupling of excited states. In addition in the absence of distinct 3D dots, strain induced vertical correlation of dots in successive layers of a multi-quantum-dot-layer sample is not possible. A promising alternative approach to induce $3 \mathrm{D}$ dot formation in this system is to combine a low temperature epitaxial growth of a few monolayers (ML) of CdSe and subsequent in-situ annealing at considerably higher temperatures [3] (method A). The thermal treatment leads to a topological transition from an essentially $2 \mathrm{D}$ layer to an ensemble of $3 \mathrm{D}$ dot like features atop an undulated surface. In this work we have investigated systematically the effect of various growth and annealing parameters of this process on the morphological and optical properties of the resultant dots. A comparison of the properties of these dots with those grown by conventional MBE (method B) at $300^{\circ} \mathrm{C}$ reveals an order of magnitude lower dot density in case of the former. Unlike for MBE-grown samples the surface morphology shows isolated dot like features, 1-2 nm high. Based on the experimental observations we present a model of QD growth by this process which explains qualitatively the optical and structural properties of the dots.

\section{Experiments}

All samples were grown in a Riber 2300 MBE chamber equipped with elemental Zn, Cd, and Se effusion cells. Prior to the growth of the II-VI layers, an undoped GaAs buffer, $200 \mathrm{~nm}$ thick, was grown atop the oxide-desorbed, epi-ready GaAs:Si (100) substrates, in another inter-connected Riber 2300 MBE chamber. The typical steps in the growth of the samples are as follows: A ZnSe buffer, $45-50 \mathrm{~nm}$ thick, is first grown at $280^{\circ} \mathrm{C}$. The sample is then cooled down to $230^{\circ} \mathrm{C}$ under $\mathrm{Zn}$ flux and a few monolayers of CdSe are grown by conventional MBE. Immediately afterwards, the sample is heated up to temperatures between $280-340^{\circ} \mathrm{C}$ under Se flux, and annealed for 20 minutes. This is followed by cooling the sample down to $280^{\circ} \mathrm{C}$ and subsequent MBE growth of a 30-50 nm thick ZnSe cap layer. The typical structures of the samples grown by MBE are also similar. However, the growth of all layers was carried out at $300^{\circ} \mathrm{C}$, and no growth interruption was introduced at any step. High resolution X-ray diffraction (HRXRD) was carried out with a 4 -crystal diffractometer using a $\mathrm{Cu} K_{\alpha}$ source. Atomic force microscopy 
(AFM) was performed in contact mode, in atmospheric ambient, typically within 60-90 min after the growth of the samples. Photoluminescence (PL) spectra were recorded at $1.6 \mathrm{~K}$, using an $\mathrm{Ar}^{+}$laser (UV multiple line), a $1 \mathrm{~m}$ spectrometer with $1200 \mathrm{~mm}^{-1}$ grating, and a $\mathrm{LN}_{2}$ cooled CCD camera. For micro-PL measurements, mesas with lateral dimensions down to $50 \mathrm{~nm}$ were fabricated using e-beam lithography and chemically assisted ion beam etching. Raman scattering was excited using blue/green $\mathrm{Ar}^{+}$laser lines and was detected by a $0.85 \mathrm{~m}$ double spectrometer with a Si multichannel detector.

\section{Results}

To determine, and thereby calibrate, the amount of CdSe within the samples, a powerful technique known as X-ray interferometry (XRI) was employed. An epitaxial structure, wherein a thin layer of composition A is sandwiched between two thick cladding layers of composition B, in our case CdSe and ZnSe, respectively, serves as a Bragg-case interferometer for short wavelength X-rays, typically used for X-ray crystallography. Due to the different lattice constants of CdSe and ZnSe, a phase shift is introduced between the X-ray beams diffracted from the ZnSe buffer and cap layers, resulting in a further modulation of the pendellösung fringes in the recorded diffractograms. A careful simulation of the experimental diffraction patterns enables a precise determination of the amount of CdSe within the sample. An advantage of using this technique is in its insensitivity to the distribution of the CdSe within the ZnSe matrix as long as plastic deformation does not set in. The bottom-most profile of Fig. 1 (discussed in detail later) is a typical example. In the inset, the recorded diffraction pattern is compared to

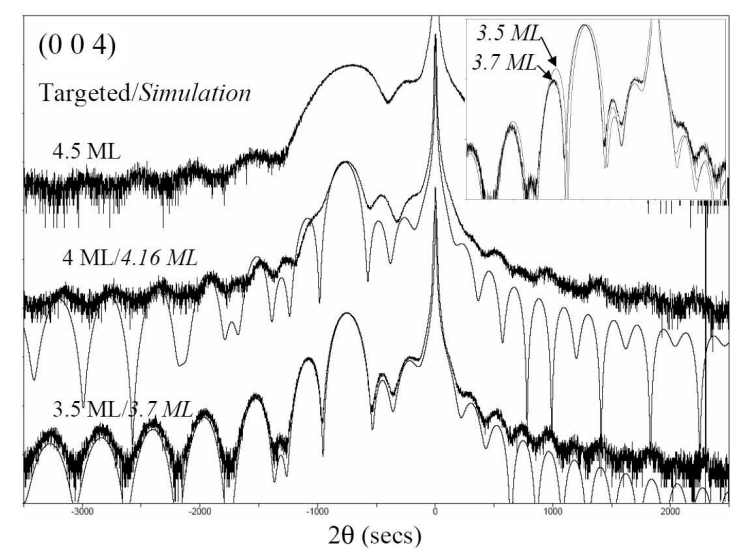

Fig. 1. Diffractograms of (004) reflections from samples grown by method A with nominal CdSe coverages of 3.5, 4, and 4.5 ML, along with the simulated profiles (except for 4.5 ML CdSe). Inset shows an enlarged section of the bottom-most recorded profile and two simulated profiles with 3.5 ML and 3.7 ML CdSe. 
two simulated profiles for 3.5 and 3.7 ML CdSe coverage. It illustrates that even sub-monolayer variations in CdSe coverage can be clearly detected.

To start with, a series of samples with varying CdSe coverages was grown by both methods $\mathrm{A}\left(\mathrm{MBE}\right.$ at $230^{\circ} \mathrm{C}$ and in-situ annealing at $310^{\circ} \mathrm{C}$ ) and method $\mathrm{B}$ $\left(\mathrm{MBE}\right.$ at $\left.300^{\circ} \mathrm{C}\right)$. Possible errors in interpreting experimental results, due to uncertainty in the CdSe content, could be eliminated since XRI enabled an accurate determination of the amount of CdSe and an apriori calibration of the growth rate, both at $230^{\circ} \mathrm{C}$ and $300^{\circ} \mathrm{C}$. The diffractograms shown in Fig. 1 are for samples grown by method A, with nominal CdSe contents of 3.5 , 4, and $4.5 \mathrm{ML}$, respectively. Up to $3.5 \mathrm{ML}$ CdSe coverage, a good agreement is evident between the measured and the simulated profiles and the calculated CdSe coverages concur well to the values targeted. This also indicates that despite the long annealing durations in method $\mathrm{A}$, no significant desorption of $\mathrm{Cd}$ takes place. Beyond 3.5 ML CdSe, the recorded pattern begins to deviate from the simulated one, and the fringes tend to overlap increasingly. This evolution can be attributed to the generation of stacking faults [4].

Figure $2 \mathrm{a}$ and $\mathrm{b}$ shows the AFM images of two uncapped samples with $3 \mathrm{ML}$ CdSe content, grown by methods A and B, respectively. The topology of CdSe deposited by method B shows a RMS roughness of $0.5 \mathrm{~nm}$, attributable primarily to the roughness of the underlying ZnSe buffer layer. The presence of tiny features, with lateral separations below $\sim 10 \mathrm{~nm}$, cannot be ruled out as they would not be distinguishable due to the resolution limit of AFM. The sample grown by method $\mathrm{A}$, in contrast shows discernible dot-like features atop such an undulated surface. The 3D island features are 1-2 nm high, about $20 \mathrm{~nm}$ in diameter and with a density of $6 \times 10^{10} \mathrm{~cm}^{-2}$.
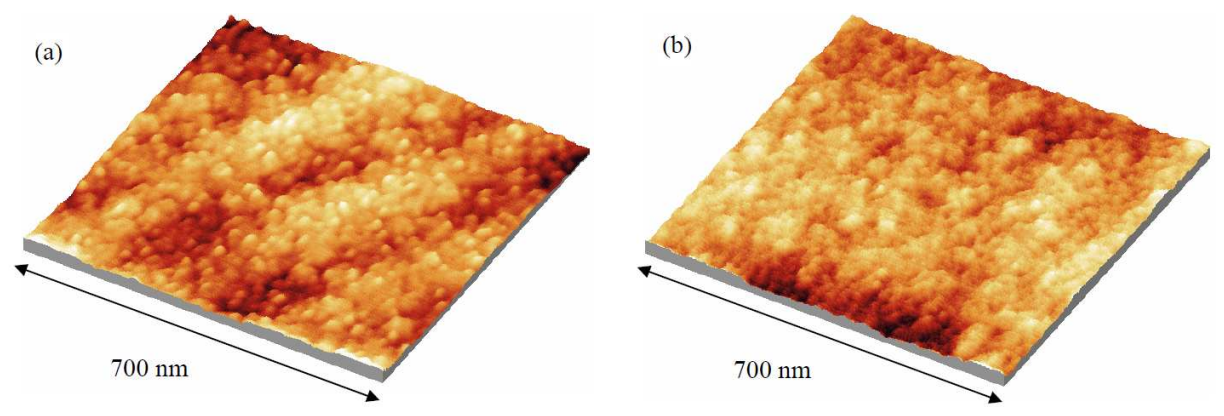

Fig. 2. AFM images of samples with $3 \mathrm{ML}$ CdSe, grown by (a) method A (height scale: $5 \mathrm{~nm}$ ) and (b) method B (height scale: $4 \mathrm{~nm}$ ).

The dot densities for both methods were also determined by micro-PL measurements. Figure $3 \mathrm{a}$ and $\mathrm{b}$ shows the spectra recorded from samples with $3 \mathrm{ML}$ CdSe, grown by method A and B, respectively. Each single line is assigned to discrete exciton (or biexciton) states. Their number is considerably smaller for 

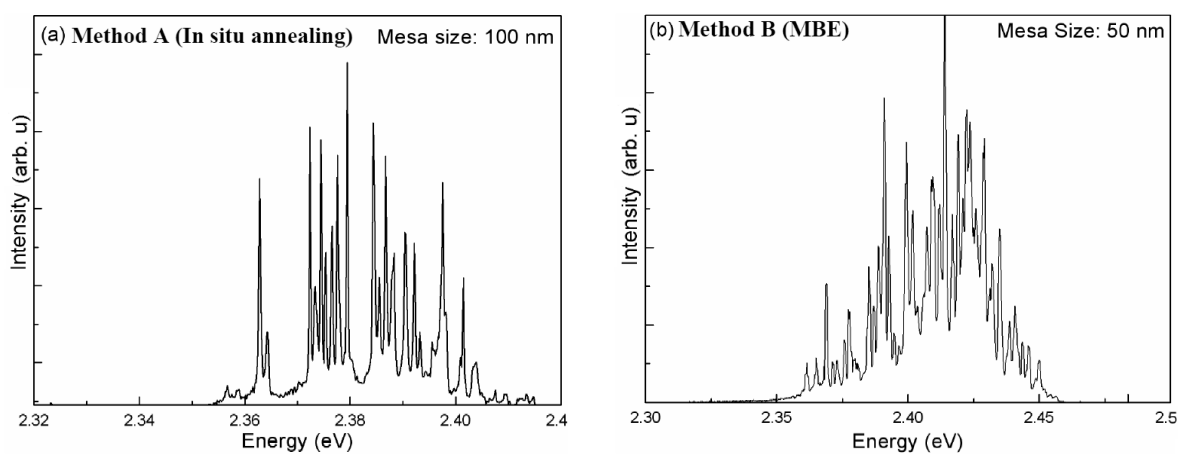

Fig. 3. Micro-photoluminescence spectra of samples with $3 \mathrm{ML}$ CdSe, grown by (a) method A and (b) method B. The mesa sizes are $100 \mathrm{~nm}$ and $50 \mathrm{~nm}$, respectively.

method A compared to method B, albeit the mesa size is four times larger. The calculated densities of dots are in the range of $10^{11} \mathrm{~cm}^{-2}$ and $10^{12} \mathrm{~cm}^{-2}$ for methods A and B, respectively.

Figure 4 shows the macroscopic PL spectra for both series of samples, recorded at $1.6 \mathrm{~K}$. Despite long growth interruptions during annealing, no significant loss of luminescence intensity is observed in comparison to the samples grown by method B. However, for both methods, at around 4 ML CdSe, the intensity drops notably. This can be ascribed to the formation of dislocations within the larger dots for higher CdSe coverages, in agreement with the overlap of pendellösung fringes in HRXRD for coverages beyond 3.5 ML. Interestingly, the luminescence energies for both methods show a similar monotonic decrease with increasing amounts of CdSe and also nearly the same values for all coverages. If we assume that the structures resulting from conventional MBE are essentially rough quantum wells with spatial variations in effective CdSe layer thickness and Cd concentration [5], this observation is counter-intuitive, as one would expect for method A, a drastic PL-red-shift beyond a definite CdSe coverage indicating
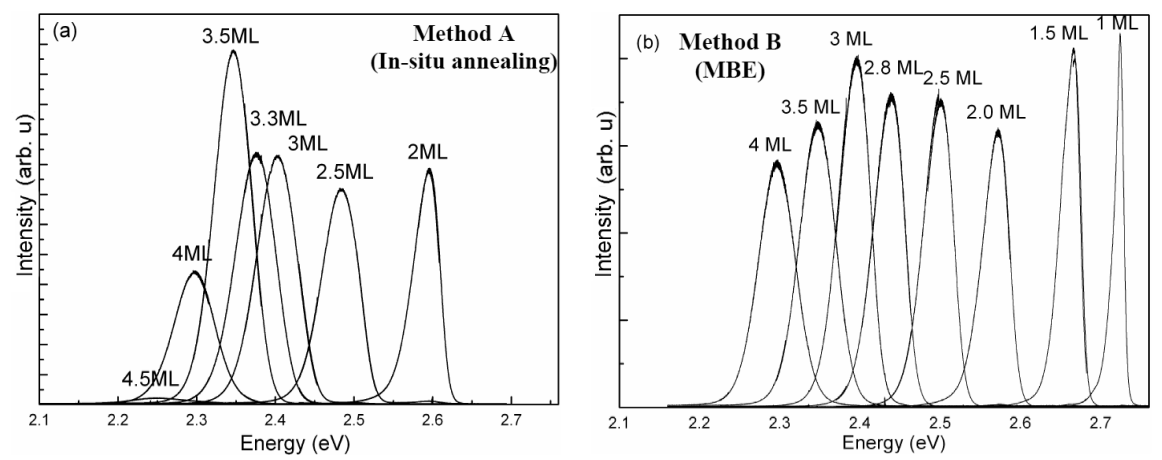

Fig. 4. Photoluminescence spectra, recorded at $1.6 \mathrm{~K}$, for samples with different nominal CdSe coverages, grown by (a) method A and (b) method B, respectively. 


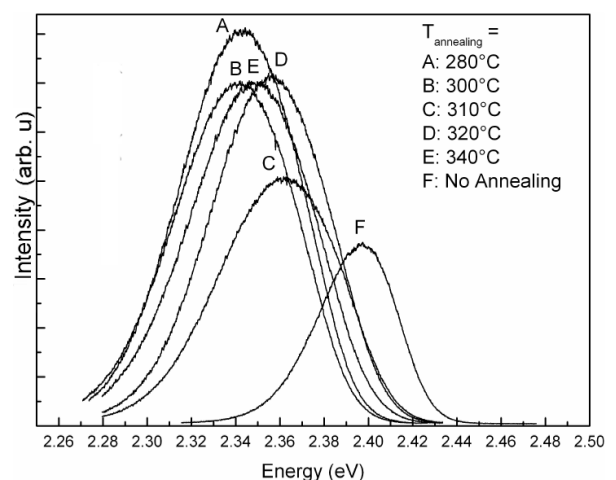

Fig. 5. Photoluminescence spectra, recorded at $1.6 \mathrm{~K}$, for a series of method A-grown samples, annealed for $20 \mathrm{~min}$ at different temperatures. A spectrum from a quasi-2D layer grown and immediately capped at $230^{\circ} \mathrm{C}$ is also shown. The nominal CdSe layer thickness for all samples is 3.3 ML.

3D dot formation. This is well known for S-K dots, i.e. InAs dots on GaAs [6]. In order to understand this parallelism of PL-red-shift trends and to study further the phenomenon of dot formation by this technique, we varied, in a series of samples with $3.3 \mathrm{ML} \mathrm{CdSe}$, the annealing temperature $T_{\text {anneal }}$, between $280^{\circ} \mathrm{C}$ and $340^{\circ} \mathrm{C}$. Figure 5 shows the PL spectra of the samples, along with that of a quasi-2D layer grown and immediately capped at $230^{\circ} \mathrm{C}$. Compared to the spectrum corresponding to this layer, spectra from all other annealed layers are significantly red-shifted. The maximum red-shift of about $80 \mathrm{meV}$ is observed for $T_{\text {anneal }}=280^{\circ} \mathrm{C}$ and $300^{\circ} \mathrm{C}$. This certainly suggests some topological transition leading to $3 \mathrm{D}$ dot formation. Comparing this red-shift with the confinement energies in thin CdSe/ZnSe quantum wells, we evaluated the effective dot height to be as small as $1-1.5 \mathrm{~nm}$. This value is in reasonable agreement with the AFM measurements (Fig. 1a) and indicates that in-situ annealing induces 3D dot formation by the redistribution of an amount of CdSe corresponding only to a fraction of a ML. These dots are atop a rough residual layer that represents a continuum of 2D-like states. Resonant enhancement of Raman scattering by longitudinal optical (LO) phonons with green/blue laser excitation allowed us to observe strong signals even from a single CdSe dot layer. From the frequency shift of the ZnCdSe phonon (due to alloying and biaxial strain), calibrated with a pseudomorphic $\mathrm{Zn}_{0.7} \mathrm{Cd}_{0.3} \mathrm{Se}$ layer on $\mathrm{ZnSe}$, a high $\mathrm{Cd}$ content, increasing with increasing CdSe coverage, was deduced for the samples. For 4 ML nominal coverage, the results indicate that the core of the dots consists of pure CdSe. This proves that intermixing during deposition, as well as the long periods of annealing, are minimal and limited only to a ML length scale.

Several of our experimental observations from samples grown by method A, i.e., the gradual PL-red-shift with increasing CdSe coverage, the redistribution of only a limited amount of CdSe during QD formation for all CdSe coverages, and 
the marginal influence of annealing temperature on the dot properties contradict the results known for the III-V and IV-VI dots, self organized as a result of the strain induced S-K transition. The formation of dots by method A can be understood by the following model, proposed in part by Kratzert [7]: MBE deposition of CdSe, both at $230^{\circ} \mathrm{C}$ and $300^{\circ} \mathrm{C}$, results in an atomically rough layer consisting of $1 \mathrm{ML}$ high 2D islands, mobile adatoms, and singly-bonded edge-atoms. The roughness at $230^{\circ} \mathrm{C}$, however, is expected to be of larger lateral scale, compared to that at $300^{\circ} \mathrm{C}$, with lower density of $2 \mathrm{D}$ islands and mobile adatoms. This is because the growth at this temperature proceeds in a layer-by-layer mode, in contrast to that at $300^{\circ} \mathrm{C}$, known from RHEED intensity oscillation profiles [8]. Additionally, annealing the layer at $230^{\circ} \mathrm{C}$ for a considerable time results in a flattening of the layer [9]. This suggests that mobile adatoms at $230^{\circ} \mathrm{C}$ may climb down a $2 \mathrm{D}$ island edge but climbing up, and thereby forming $3 \mathrm{D}$ islands is restricted due to the larger thermal activation energy required for this process. The up-climb of mobile-adatoms can however, be enhanced (a) by an immediate increase in temperature after growth or (b) by growing the CdSe layer at a higher temperature. The former represents method $\mathrm{A}$ and the latter method B, in our case. The ratio of the up- to down-climb rates, $R_{\uparrow} / R_{\downarrow}$, increases exponentially with substrate temperature. Thus, with temperature ramp-up immediately after the growth in method $\mathrm{A}$, the adatoms at the edges are thermally activated also to climb on top of the existing 2D islands and eventually lead to 3D dot formation. Islanding by this process, therefore, indeed involves the top fractional monolayer of CdSe, and is not strain induced in the S-K sense. Rather, the important determinants of the island formation in this essentially thermally activated process are (a) the concentration of mobile adatoms, (b) the concentration of $2 \mathrm{D}$ islands, and (c) the ratio of the probabilities of these atoms climbing onto and down from the $2 \mathrm{D}$ islands, $R_{\uparrow} / R_{\downarrow}$. In method B, i.e. $\mathrm{MBE}$ at $300^{\circ} \mathrm{C}$ and immediate capping as discussed before, the concentration of both mobile adatoms and $2 \mathrm{D}$ islands, which act as the nucleation centers for the 3D islands, is higher. $R_{\uparrow} / R_{\downarrow}$ is also high due to the high temperature, which means that the process ends up with a very high density of dots or essentially a rough surface. The discussion above thus explains (a) the gradual PL-red-shift and the absence of a clear critical thickness for dot formation, in essence for both methods, and (b) the reduced density of dots by method A, determined from spectrally resolved sharp emissions in micro-PL (Fig. 4). Also the observed moderate PL-red-shift with annealing and the marginal influence of annealing temperature (Fig. 5) are well understood by this model considering the fact that the amount of CdSe involved in the 3D dot formation is limited only to a fraction of a ML. The islands therefore, are indeed tiny, as observed, and also do not have a further possibility of growing in dimensions even at higher temperatures, during annealing (method A) or growth (method B). A similar effective dot height is therefore achieved by both methods, leading to very similar PL energies for all coverages (Fig. 4). 


\section{Conclusions}

The formation of CdSe on ZnSe by a technique, combining low temperature MBE and in-situ annealing was studied. In comparison to conventional MBE grown dots, these dots show a similar photoluminescence behavior but more pronounced discrete 3D morphology. The density of such 3D features is about $10^{11} \mathrm{~cm}^{-2}$, which is a decade lower compared to the MBE grown samples. QD formation by this method is described by the thermally activated redistribution of a certain amount of mobile adatoms at the CdSe surface with 2D islands acting as nucleation sites for the 3D island formation.

\section{Acknowledgments}

We thank V. Hock and E. Margapoti for the preparation of the mesa structures and for the micro-PL measurements, respectively. This work was supported by the Deutsche Forschungsgemeinschaft in the framework of SFB 410.

\section{References}

[1] N. Peranio, A. Rosenauer, D. Gerthsen, S.V. Sorokin, I.V. Sedova, S.V. Ivanov, Phys. Rev. B 61, 16015 (2000).

[2] D. Litvinov, A. Rosenauer, D. Gerthsen, H. Preis, S. Bauer, E. Kurtz, J. Appl. Phys. 89, 4150 (2001).

[3] P.R. Kratzert, M. Rabe, F. Henneberger, Phys. Status Solidi B 224, 179 (2001).

[4] T. Passow H. Heinke, J. Falta, K. Leonardi, D. Hommel, Appl. Phys. Lett. 77, 3544 (2000).

[5] T. Passow, K. Leonardi, H. Heinke, D. Hommel, D. Litvinov, A. Rosenauer, D. Gerthsen, J. Seufert, G. Bacher, A. Forchel, J. Appl. Phys. 92, 6546 (2002).

[6] J.M. Gérard, J.B. Génin, J. Lefebvre, J.M. Moison, N. Lebouché, F. Barthe, J. Cryst. Growth 150, 351 (1995).

[7] P. Kratzert, Ph.D. Thesis, Humboldt Universität, Berlin 2002, p. 60.

[8] P. Kratzert, Ph.D. Thesis, Humboldt Universität, Berlin 2002, p. 35.

[9] D. Litvinov, A. Rosenauer, D. Gerthsen, P. Kratzert, M. Rabe, F. Henneberger, Appl. Phys. Lett. 81, 640 (2002). 